\title{
A retrospective review of mortality in lorises and pottos in North American zoos, 1980-2010
}

\author{
Grace Fuller $^{1,2, *}$, Kristen E. Lukas ${ }^{1,2}$, Christopher Kuhar $^{1,2}$, Patricia M. Dennis ${ }^{1,2,3}$ \\ ${ }^{1}$ Department of Biology, Case Western Reserve University, 10900 Euclid Avenue, Cleveland, Ohio 44106, USA \\ ${ }^{2}$ Cleveland Metroparks Zoo, 3900 Wildlife Way, Cleveland, Ohio 44109, USA \\ ${ }^{3}$ Department of Veterinary Preventive Medicine, The Ohio State University, 1900 Coffey Road, Columbus, Ohio 43210, USA
}

\begin{abstract}
Patterns of mortality in captive animals can reveal potentially problematic care practices or other risk factors that may negatively impact animal health and population sustainability. We reviewed death records (necropsy and/or histopathology reports) for 367 lorises and pottos born between 1980 and 2010 that were housed in 33 North American zoos and related facilities. Our sample included 20 Loris tardigradus nordicus, 72 L. t. tardigradus, 109 Nycticebus coucang, 133 N. pygmaeus, and 33 Perodicticus potto. In all 5 groups, neonates (animals < $30 \mathrm{~d}$ old) or geriatric animals accounted for the majority of death reports. Neonate deaths were most commonly attributed to stillbirth, trauma, and multi-systemic disease (septicemia or other infection). Animals that survived the first month of life were likely to live to adulthood or the geriatric stage. For all species, the most commonly cited causes of death were renal disease, multi-systemic illness, and trauma. Over $50 \%$ of all the animals studied displayed some renal pathology upon postmortem analysis. Across species, other organ systems in which $>20 \%$ of animals showed signs of disease included the cardiovascular and hemolymphatic, endocrine and metabolic, gastrointestinal, and immunologic systems. Our results indicate that reducing neonatal mortality is a major priority for sustaining these species in captivity, and common practices for managing groups during the perinatal period should be carefully scrutinized. Other areas in need of further investigation are environmental contributions to neoplastic and infectious disease, and dietary contributions to chronic degenerative conditions.
\end{abstract}

KEY WORDS: Lorisid primate $\cdot$ Epidemiology $\cdot$ Zoo veterinary care $\cdot$ Infant mortality

\section{INTRODUCTION}

Lorises (genera Loris and Nycticebus) are strepsirrhine primates that dwell in forested habitats across Southeast Asia. Slender lorises include at least 2 species in India and Sri Lanka (Brandon-Jones et al. 2004), and the slow lorises are a radiation of species extending through Indonesia into southern China. Ongoing investigations are revealing increased diversity within the genus Nycticebus (Munds et al. 2013), just as it is becoming clear that all loris species are declining in the wild (Nekaris \& Jayewardene 2004, Nekaris \& Nijman 2007, Iseborn et al. 2011). In recent years, the pet trade has had an increasingly devastating impact on wild slow loris populations (Nekaris et al. 2010, Nekaris \& Campbell 2012). Many animals rescued from this illegal trade have suffered physical injuries that make reintroduction to the wild impossible, and the need for sanctuary housing grows along with the popularity of lorises as pets (Nekaris \& Jaffe 2007). Zoos that have historically housed lorises thus may have the opportunity to contribute to in situ conservation efforts by providing sanctuaries with information on captive loris husbandry and veterinary care.

Zoo populations of slender and slow lorises are found in Europe, Asia, and North America. North American zoos have historically housed the pygmy 
slow loris Nycticebus pygmaeus, and a hybridized population of $N$. coucang, $N$. bengalensis, and $N$. javanicus, all managed as $N$. coucang (common name: slow loris). North American zoos have also housed 2 subspecies of red slender loris: Loris tardigradus tardigradus and L. t. nordicus, although the North American captive population of the latter is now extinct. According to Fitch-Snyder \& Schulze (2001), the history of slow and slender lorises in North America dates back to late 19th/early 20th century exhibits at the Philadelphia and Bronx zoos, while a breeding population of pygmy slow lorises was established in North America in 1987. In addition to slender and slow lorises, North American zoos are home to a small population of an African lorisid: the potto Perodicticus potto. Pottos are thought to be widespread throughout sub-Saharan Africa, but their true taxonomic and conservation status is largely unknown (Grubb et al. 2003).

Pygmy loris numbers are increasing in zoos, and currently they are the only lorisid species nearing sustainability in North American collections (Kuhar et al. 2011). Populations of other lorisid species are small (<15 ind.) and declining. Although L. t. nordicus is no longer found in captivity in North America, there are still some individuals managed in Europe. Research into reproduction, as well as veterinary and social needs, may play an important role in maintaining viable populations of these species in captivity (Schulze 1998).

High infant mortality and traumatic death appear to be major impediments to population growth in lorises and pottos. Debyser (1995) analyzed mortality trends for strepsirrhine juveniles in zoos and primate centers and found that more offspring died prior to weaning in N. coucang, L. t. tardigradus, and P. potto than the 13 lemur and galago species that were also surveyed. Sutherland-Smith \& Stalis (2001) reviewed mortality in lorises from the San Diego Zoo (1982 to 1995) compared to Duke University Lemur Center (1980 to 1994). Those data showed that in addition to trauma, significant contributors to captive loris morbidity and mortality include dental, renal, and respiratory diseases. The present study expands on this research (with some sampling overlap) and seeks to describe more comprehensively the factors contributing to mortality in captive lorises and pottos.

We reviewed postmortem records for $L$. t. nordicus, L. t. tardigradus, N. coucang, N. pygmaeus, and $P$. potto born between 1980 and 2010 that lived in North American zoos. Our primary aim was to describe major sources of mortality in these species. In a companion to the present paper, we also surveyed facili- ties that are currently housing lorisid primates to determine how animals are actually cared for in practice (Fuller et al. 2013). These studies set the stage for future hypothesis-driven research examining associations between environmental design, diet, health, and animal behavior. Ultimately, our goal is to identify husbandry concerns and areas of research most urgently needed to address the health, welfare, and population sustainability of lorises and pottos in the care of sanctuaries and zoos.

\section{MATERIALS AND METHODS}

\section{Study population}

We requested full medical records from all North American zoos and related facilities for animals born between January 1, 1980 and December 31, 2010 representing 5 species: Loris tardigradus nordicus, L. $t$. tardigradus, Nycticebus coucang, N. pygmaeus, and Perodicticus potto. Animals and facilities included in these populations were identified by consulting North American Regional Studbooks for each species as of October 2010. Species groups are also based on studbook data. Studbooks were accessed using Poplink v. 1.3 and 2.1. L. t. nordicus and L. t. tardigradus have separate studbooks and were treated as separate populations here. In contrast, $N$. coucang, $N$. bengalensis, and $N$. javanicus have historically been grouped as one species in North American facilities, and the population's hybrid status is uncertain; for these reasons, they are treated as a single population (referred to as $N$. coucang) here.

In total, 1 unaccredited zoo and 32 of 35 zoos (91\%) accredited by the Association of Zoos and Aquariums (AZA) where a lorisid primate died during this time period contributed medical records to this study, representing a total of 367 animals. The total number of records received for each species were: 20 (10:9:1 (male:female:unknown) out of 25 L.t. nordicus (80\%), $72(31: 29: 12)$ out of 80 L. t. tardigradus $(90 \%), 109$ (50:52:7) out of $152 \mathrm{~N}$. coucang (72\%), 133 (59:57:17) out of $1731 \mathrm{~N}$. pygmaeus (77\%), and 33 (17:11:5) of 37 P. potto $(79 \%)$. Across species, this sample included 167 males $(45.5 \%), 158$ females $(43.0 \%)$, and 42 animals of unknown sex $(11.4 \%)$.

The majority of records $(241 ; 66 \%)$ were formatted using MedARKS or ARKS (International Species Information Systems), but the sample included other electronic formats and hand-written records. Additionally, one facility elected to provide summaries of necropsy and histopathology findings rather than 
submitting full records. Medical records included in this study varied greatly in detail and scope. Necropsy reports were available in 247 (67\%) of the records received, and 214 (58\%) included a histopathology report.

Independent samples' $t$-tests with equal variances assumed were used to compare mean age at death for males compared to females within each species studied (IBM SPSS Statistics v. 21).

\section{Organ system classification and cause of death}

Data for each animal were entered into a Microsoft Access database. Age at death, sex, and death type (spontaneous, euthanasia, or unknown) were recorded for each animal. Based on their age at death $(x)$, the animals within each species were categorized into 1 of 4 age groups: neonate: $x \leq 30 \mathrm{~d}$ old; juvenile: $30 \mathrm{~d}<\mathrm{x} \leq 1 \mathrm{yr}$; adult: $1 \mathrm{yr}<\mathrm{x} \leq$ geriatric; and geriatric. Age at which an animal was considered geriatric varied by species and was calculated as $75 \%$ of the mean lifespan of all animals in our study population that reached adulthood (i.e. excluding neonates and juveniles). L. $t$. nordicus were considered geriatric at $9 \mathrm{yr}$ old, L. t. tardigradus at $10 \mathrm{yr}, N$. coucang at $9 \mathrm{yr}, N$. pygmaeus at $8 \mathrm{yr}$, and $P$. potto at $12 \mathrm{yr}$. One year was chosen as a common cutoff point between juveniles and adults based on published reports of age at first reproduction in these species (Charles-Dominique 1977, Fitch-Snyder \& Schulze 2001).

Diagnoses from necropsy and histopathology reports were used to determine which organ systems showed evidence of pathology at the time of the animal's death. Organ system classifications were based on Hope \& Deem (2006) and included the following categories: cardiovascular and hemolymphatic; central and peripheral nervous system (CNS); dental; endocrine and metabolic; ear, nose, and throat (ENT); gastrointestinal; hepatic and biliary; immunologic (including lymph nodes); integumentary; musculoskeletal; ocular; renal (including the urinary bladder); reproductive; respiratory; and whole body.

Records were also reviewed to determine the cause of each animal's death or primary reason for being euthanized. If a cause of death was not clearly indicated in the medical report, one was identified based on organ system pathology described in the record. In addition to the organ systems listed above (see previous paragraph), cause of death included the following categories specific to neonates: maternal neg- lect; stillborn and abortion; and unexplained neonate death. Deaths for neonates were considered unexplained when no diagnosis was given, whether or not a necropsy and/or histopathology exam was performed (and available in the record). For all other age classes, the cause of death was only listed as unknown if the record contained a necropsy and histopathology report but a primary cause of death was still unclear. When a histopathology report was not available and the cause of death was not clearly stated in the record, the cause of death was undeterminable and was listed as an incomplete record. For all age classes, in clear cases of environmental or social injury, the cause of death was listed as trauma, even if death was more immediately attributable to septicemia or infection subsequent to the initial insult.

\section{RESULTS}

\section{Age-specific patterns of mortality}

For all animals in the present study, mean age of death, regardless of death type, was 7.7 yr (SD: $7.1 \mathrm{yr}$ ). The mean age at death for each species was: $8.9 \mathrm{yr}$ (SD: 6.6, range: 0 to $18.7 \mathrm{yr}$ ) for $L$. $t$. nordicus, $8.0 \mathrm{yr}$ (SD: 7.3, range: 0 to $21.5 \mathrm{yr}$ ) for $L$. $t$. tardigradus, $9.5 \mathrm{yr}$ (SD: 6.8, range: 0 to $24.3 \mathrm{yr}$ ) for $N$. coucang, 6.4 yr (SD: 6.3, range: 0 to $21.9 \mathrm{yr}$ ) for $N$. pygmaeus, and $6.2 \mathrm{yr}$ (SD: 9.0, range: 0 to $25.7 \mathrm{yr}$ ) for $P$. potto. Taking into account only animals that reached adulthood, the mean age at death for each species was: $11.8 \mathrm{yr}(\mathrm{SD}: 4.7 \mathrm{yr}, \mathrm{n}=15$ ) for $L$. $t$. nordicus, $12.7 \mathrm{yr}(\mathrm{SD}: 5.1 \mathrm{yr}, \mathrm{n}=45)$ for $L$. $t$. tardigradus, $11.8 \mathrm{yr}(\mathrm{SD}: 5.6 \mathrm{yr}, \mathrm{n}=88)$ for $N$. coucang, $10.1 \mathrm{yr}(\mathrm{SD}: 5.1 \mathrm{yr}, \mathrm{n}=84$ ) for $N$. pygmaeus, and 15.6 yr (SD: $7.3 \mathrm{yr}, \mathrm{n}=13$ ) for $P$. potto. Mean age at death for individuals that reached adulthood was nearly equal for males and females in every species tested, and there were no significant differences in lifespan based on sex.

For all species except $L$. $t$. nordicus, death most commonly occurred spontaneously (Table 1) rather than as a result of euthanasia. Neonate mortality (death within the first $30 \mathrm{~d}$ of life) was high for each species, most strikingly the potto, in which $60.6 \%$ of the sample population died by $30 \mathrm{~d}$ of age (Table 2 ). Of all the animals in this study, 30.2\% (111/367) died as neonates. For all species, mortality during the juvenile period was relatively low. Generally, mortality was greatest in neonate and geriatric groups (Table 2). 
Table 1. Recorded type of death for lorises and pottos in North American facilities, 1980-2010. Data are \% of total for each species or for all species, followed by number of individuals in parentheses. N: total sample size

\begin{tabular}{|lcccccc|}
\hline Death type & $\begin{array}{c}\text { Loris tardigradus } \\
\text { nordicus } \\
\mathrm{N}=20\end{array}$ & $\begin{array}{c}\text { Loris tardigradus } \\
\text { tardigradus } \\
\mathrm{N}=72\end{array}$ & $\begin{array}{c}\text { Nycticebus } \\
\text { coucang } \\
\mathrm{N}=109\end{array}$ & $\begin{array}{c}\text { Nycticebus } \\
\text { pygmaeus } \\
\mathrm{N}=133\end{array}$ & $\begin{array}{c}\text { Perodicticus } \\
\text { potto }\end{array}$ & $\begin{array}{c}\text { Total } \\
\mathrm{N}=33\end{array}$ \\
$\mathrm{~N}=367$ \\
Euthanasia & $45.0(9)$ & $29.2(21)$ & $30.3(33)$ & $30.1(40)$ & $21.2(7)$ & $30(110)$ \\
Spontaneous & $40.0(8)$ & $69.4(50)$ & $63.3(69)$ & $67.7(90)$ & $69.7(23)$ & $65.4(240)$ \\
Unknown & $15.0(3)$ & $1.4(1)$ & $6.4(7)$ & $2.2(3)$ & $9.1(3)$ & $4.6(17)$ \\
\hline
\end{tabular}

Table 2. Distribution of loris and potto deaths by age class in North American facilities, 1980-2010. Data are \% of total for each species or for all species, followed by number of individuals in parentheses. N: total sample size; $x$ : age at death

\begin{tabular}{|c|c|c|c|c|c|c|}
\hline Age class & $\begin{array}{l}\text { Loris tardigradus } \\
\text { nordicus } \\
\mathrm{N}=20\end{array}$ & $\begin{array}{l}\text { Loris tardigradus } \\
\text { tardigradus } \\
\mathrm{N}=72\end{array}$ & $\begin{array}{l}\text { Nycticebus } \\
\text { coucang } \\
\mathrm{N}=109\end{array}$ & $\begin{array}{c}\text { Nycticebus } \\
\text { pygmaeus } \\
\mathrm{N}=133\end{array}$ & $\begin{array}{c}\text { Perodicticus } \\
\text { potto } \\
\mathrm{N}=33\end{array}$ & $\begin{array}{c}\text { Total } \\
\mathrm{N}=367\end{array}$ \\
\hline $\begin{array}{l}\text { Neonate } \\
(x \leq 30 \mathrm{~d})\end{array}$ & $25.0(5)$ & 31.9 (23) & $15.6(17)$ & $34.6(46)$ & $60.6(20)$ & $30.2(111)$ \\
\hline $\begin{array}{l}\text { Juvenile } \\
\qquad(30 \mathrm{~d}<x \leq 1 \mathrm{yr})\end{array}$ & 0 & $5.6(4)$ & $3.7(4)$ & $2.3(3)$ & 0 & $5.2(19)$ \\
\hline $\begin{array}{l}\text { Adult } \\
\qquad\left(1 \mathrm{yr}<x \leq \text { geriatric }^{\mathrm{a}}\right)\end{array}$ & $20.0(4)$ & $15.3(11)$ & $24.8(27)$ & $21.1(28)$ & $9.1(3)$ & $17.7(65)$ \\
\hline Geriatric & $55.0(11)$ & $47.2(34)$ & $56.0(61)$ & $42.1(56)$ & $30.3(10)$ & $46.9(172)$ \\
\hline
\end{tabular}

Table 3. Primary cause of death or reason for euthanasia in lorises and pottos housed in North American facilities, $1980-2010$. Data are \% of total for each species or for all species, followed by number of individuals in parentheses. N: total sample size, followed by number of individuals that were male:female:unknown in parentheses

\begin{tabular}{|c|c|c|c|c|c|c|}
\hline Cause of death & $\begin{array}{l}\text { Loris tardigradus } \\
\text { nordicus } \\
\mathrm{N}=20 \\
(10: 9: 1)\end{array}$ & $\begin{array}{c}\text { Loris tardigradus } \\
\text { tardigradus } \\
\mathrm{N}=72 \\
(31: 29: 12)\end{array}$ & $\begin{array}{l}\text { Nycticebus } \\
\text { coucang } \\
\mathrm{N}=109 \\
(50: 52: 7)\end{array}$ & $\begin{array}{c}\text { Nycticebus } \\
\text { pygmaeus } \\
\mathrm{N}=133 \\
(59: 57: 17)\end{array}$ & $\begin{array}{c}\text { Perodicticus } \\
\text { potto } \\
\mathrm{N}=33 \\
(17: 11: 5)\end{array}$ & $\begin{array}{c}\text { Total } \\
\\
\mathrm{N}=367 \\
(167: 158: 42)\end{array}$ \\
\hline $\begin{array}{l}\text { Cardiovascular and } \\
\text { hemolymphatic }\end{array}$ & 0 & $4.2(3)$ & $3.7(4)$ & $4.5(6)$ & $6.1(2)$ & $4.1(15)$ \\
\hline Central nervous system & 0 & $2.8(2)$ & 0 & $2.3(3)$ & $3.0(1)$ & $1.6(6)$ \\
\hline Endocrine and metabolic & 0 & 0 & $2.8(3)$ & $0.8(1)$ & 0 & $1.1(4)$ \\
\hline Ear, nose, and throat & 0 & 0 & $2.8(3)$ & $0.8(1)$ & 0 & $1.1(4)$ \\
\hline Gastrointestinal & $5.0(1)$ & 0 & $6.4(7)$ & $1.5(2)$ & 0 & $2.7(10)$ \\
\hline Hepatic and biliary & $5.0(1)$ & $5.6(4)$ & $5.5(6)$ & $3.0(4)$ & 0 & $4.1(15)$ \\
\hline Immunologic & 0 & 0 & 0 & $0.8(1)$ & 0 & $0.3(1)$ \\
\hline Integumentary & 0 & $1.4(1)$ & 0 & $1.5(2)$ & 0 & $0.8(3)$ \\
\hline Musculoskeletal & 0 & $2.8(2)$ & $2.8(3)$ & $5.3(7)$ & 0 & $3.3(12)$ \\
\hline Ocular & $5.0(1)$ & 0 & 0 & $0.8(1)$ & 0 & $0.5(2)$ \\
\hline Renal & $25.0(5)$ & $18.1(13)$ & $19.3(21)$ & $15.0(20)$ & $6.1(2)$ & $16.6(61)$ \\
\hline Reproductive & 0 & 0 & $3.7(4)$ & $0.8(1)$ & $3.0(1)$ & $1.6(6)$ \\
\hline Respiratory & $10.0(2)$ & $4.2(3)$ & $2.8(3)$ & $5.3(7)$ & $15.2(5)$ & $5.4(20)$ \\
\hline Multi-systemic & $5.0(1)$ & $19.4(14)$ & $22.9(25)$ & $15.8(21)$ & $15.2(5)$ & $18.0(66)$ \\
\hline Trauma & $5.0(1)$ & $13.9(10)$ & $10.1(11)$ & $10.5(14)$ & $9.1(3)$ & $10.6(39)$ \\
\hline Unknown & 0 & 0 & $1.8(2)$ & $0.8(1)$ & 0 & $0.8(3)$ \\
\hline Incomplete record & $20.0(4)$ & $4.2(3)$ & $5.5(6)$ & $7.5(10)$ & $6.1(2)$ & $6.8(25)$ \\
\hline $\begin{array}{l}\text { Maternal neglect } \\
\text { (neonates) }\end{array}$ & $10.0(2)$ & $1.4(1)$ & $0.9(1)$ & $6.8(9)$ & $6.1(2)$ & $4.1(15)$ \\
\hline $\begin{array}{l}\text { Stillborn or abortion } \\
\text { (neonates) }\end{array}$ & 0 & $4.2(3)$ & $5.5(6)$ & $9.8(13)$ & $12.1(4)$ & $7.1(26)$ \\
\hline $\begin{array}{l}\text { Unexplained neonate } \\
\text { death (neonates) }\end{array}$ & $10.0(2)$ & $18.1(13)$ & $3.7(4)$ & $6.8(9)$ & $18.1(6)$ & $9.3(34)$ \\
\hline
\end{tabular}




\section{Organ system pathology and cause of death}

For all the lorises and pottos in this study, multisystemic disease was the most frequent cause of death or euthanasia, followed by renal disease and trauma (Table 3). Many animals were stillborn or died as neonates due to maternal neglect or other unexplained reasons. Only 2 to $6 \%$ of deaths were attributed to diseases in each of the cardiovascular and hemolymphatic; gastrointestinal; hepatic and biliary; musculoskeletal; and respiratory systems. Less than $2 \%$ of deaths were attributed to each of the remaining organ systems.

For all animals (Table 4), the most common organ system showing lesions or abnormalities at death was the renal system, with $50 \%$ of all animals in the study affected. Over $40 \%$ of all lorisids sampled also showed pathologies in the respiratory and hepatic and biliary systems. Across species, organ systems in which $>20 \%$ of animals showed signs of disease included the cardiovascular and hemolymphatic; endocrine and metabolic; gastrointestinal; and immunologic systems. Specific diseases affecting $>20 \%$ of animals in each species are detailed in the following subsections.

Neoplasia was responsible for the deaths of $10.6 \%$ (39/367) of animals; all of these cases occurred in pygmy and slow lorises, with the exception of 1 potto (Table 5). Medical records included 81 reports of distinct neoplastic growths in 66 animals; 39 of these were fatal. All neoplasms occurred in geriatric animals, with the exception of 2 adult $N$. coucang and 4 adult $N$. pygmaeus. Both neoplasias reported for $L$. $t$. nordicus occurred in males, as did 5 of 6 cases in L. $t$. tardigradus. Neoplasia occurred equally between the sexes in $N$. pygmaeus (13 cases in males and 14 in females) and Perodicticus potto (1 male and 1 female), but was more common in female $N$. coucang ( 20 cases) than male ( 9 cases).

Most animals that experienced traumas were neonates that died as a result of bite wounds (Table 6). Only cases in which cannibalism clearly occurred prior to death were counted as traumas. Often, cases were classified as unexplained neonate deaths because the carcass was scavenged, but it was not possible to determine if this occurred pre- or postmortem. Several cases in which conspecific bite wounds led to necrosis, cellulitis, or other infection occurred, most commonly in Nycticebus spp. All traumas thought to be related to mortality, even if cause of death was listed under another organ system, are detailed in Table 6.

\section{Loris tardigradus nordicus}

The primary cause of death was attributed to renal pathology in the majority of the L. t. nordicus in this study (Table 3). Records refer to renal failure (2/5) or glomerulonephritis, nephritis, and general glomeru-

Table 4. Percent of lorises and pottos with pathology diagnosed by organ system upon postmortem examination in North American facilities, 1980-2010. Data are \% of total for each species or for all species, followed by number of individuals in parentheses. N: total sample size

\begin{tabular}{|c|c|c|c|c|c|c|}
\hline Organ system & $\begin{array}{l}\text { Loris tardigradus } \\
\text { nordicus } \\
\mathrm{N}=20\end{array}$ & $\begin{array}{l}\text { Loris tardigradus } \\
\text { tardigradus } \\
\mathrm{N}=72\end{array}$ & $\begin{array}{l}\text { Nycticebus } \\
\text { coucang } \\
\mathrm{N}=109\end{array}$ & $\begin{array}{c}\text { Nycticebus } \\
\text { pygmaeus } \\
\mathrm{N}=133\end{array}$ & $\begin{array}{c}\text { Perodicticus } \\
\text { potto } \\
\mathrm{N}=33\end{array}$ & $\begin{array}{c}\text { Total } \\
\mathrm{N}=367\end{array}$ \\
\hline $\begin{array}{l}\text { Cardiovascular and } \\
\text { hemolymphatic }\end{array}$ & $30.0(6)$ & $26.4(19)$ & $33.0(36)$ & $32.3(43)$ & $18.2(6)$ & $30(110)$ \\
\hline Central nervous system & $20.0(4)$ & $16.7(12)$ & $15.6(17)$ & $16.5(22)$ & $18.2(6)$ & $16.6(61)$ \\
\hline Dental & $5.0(1)$ & $4.2(3)$ & $4.6(5)$ & $3.0(4)$ & 0 & $3.5(13)$ \\
\hline Endocrine and metabolic & $15.0(3)$ & $12.5(9)$ & $35.8(39)$ & $19.5(26)$ & $18.2(6)$ & $22.6(83)$ \\
\hline Ear, nose, and throat & $15.0(3)$ & $4.2(3)$ & $14.7(16)$ & $6.0(8)$ & $12.1(4)$ & $9.3(34)$ \\
\hline Gastrointestinal & $15.0(3)$ & $8.3(6)$ & $36.7(40)$ & $22.6(30)$ & $21.2(7)$ & $23.4(86)$ \\
\hline Hepatic and biliary & $15.0(3)$ & $47.2(34)$ & $51.4(56)$ & $42.9(57)$ & $27.3(9)$ & $43.3(159)$ \\
\hline Immunologic & $5.0(1)$ & $9.7(7)$ & $35.8(39)$ & $23.3(31)$ & $9.1(3)$ & $22.1(81)$ \\
\hline Integumentary & 0 & $6.9(5)$ & $5.5(6)$ & $7.5(10)$ & 0 & $5.7(21)$ \\
\hline Multi-systemic & $5.0(1)$ & $19.4(14)$ & $22.0(24)$ & $14.3(19)$ & $12.1(4)$ & $16.9(62)$ \\
\hline Musculoskeletal & $10.0(2)$ & $11.1(8)$ & $14.7(16)$ & $15.0(20)$ & $6.1(2)$ & $13.1(48)$ \\
\hline Ocular & $25.0(5)$ & $19.4(14)$ & $4.6(5)$ & $6.8(9)$ & $3.0(1)$ & $9.3(34)$ \\
\hline Renal & $50.0(10)$ & $41.7(30)$ & $57.8(63)$ & 51.9 (69) & $33.3(11)$ & 49.9 (183) \\
\hline Reproductive & $15.0(3)$ & $9.7(7)$ & $22.9(25)$ & $12.8(17)$ & $9.1(3)$ & $15(55)$ \\
\hline Respiratory & $25.0(5)$ & $47.2(34)$ & $46.8(51)$ & $46.6(62)$ & $30.3(10)$ & $44.1(162)$ \\
\hline Whole body & $20.0(4)$ & $12.5(9)$ & $11.0(12)$ & $17.3(23)$ & $18.2(6)$ & $14.7(54)$ \\
\hline
\end{tabular}


Table 5. Neoplasia reported for lorises and pottos in North American facilities, 1980-2010. n: number of cases for each species; $(-)$ no cases reported. Number of superscripts indicates number of cases that were identified as the primary cause of death for each cancer type

\begin{tabular}{|c|c|c|c|c|c|}
\hline Organ system & $\begin{array}{l}\text { Loris tardigradus } \\
\text { nordicus } \\
(\mathrm{n}=4)\end{array}$ & $\begin{array}{l}\text { Loris tardigradus } \\
\text { tardigradus } \\
(\mathrm{n}=6)\end{array}$ & $\begin{array}{l}\text { Nycticebus } \\
\text { coucang } \\
(\mathrm{n}=34)\end{array}$ & $\begin{array}{l}\text { Nycticebus } \\
\text { pygmaeus } \\
(\mathrm{n}=35)\end{array}$ & $\begin{array}{l}\text { Perodicticus } \\
\text { potto } \\
(\mathrm{n}=2)\end{array}$ \\
\hline $\begin{array}{l}\text { Cardiovascular and } \\
\text { hemolymphatic }\end{array}$ & - & - & - & $\begin{array}{l}\text { Sarcoma } \\
\text { (heart) }\end{array}$ & - \\
\hline $\begin{array}{l}\text { Central nervous } \\
\text { system }\end{array}$ & - & - & $\begin{array}{l}\text { Ependymal } \\
\text { (brain) }\end{array}$ & $\begin{array}{l}\text { Astrocytoma) } \\
\text { (brain stem }^{\mathrm{a}} \text { ) } \\
\text { Ependymoma } \\
\text { (cerebrum }^{\mathrm{a}} \text { ) }\end{array}$ & - \\
\hline $\begin{array}{l}\text { Endocrine and } \\
\text { metabolic }\end{array}$ & $\begin{array}{l}\text { Adenoma } \\
\text { (parathyroid) } \\
\text { Myelolipoma } \\
\text { (adrenal) }\end{array}$ & $\begin{array}{c}2 \text { adenoma } \\
\text { (1 adrenal, } \\
1 \text { thyroid) }\end{array}$ & $\begin{array}{l}\text { Adenoma } \\
\text { (adrenal) } \\
\text { Carcinoma } \\
\left(\text { pancreas }^{a}\right)\end{array}$ & $\begin{array}{l}\text { Adenoma } \\
\text { (thyroid) } \\
\text { Sarcoma } \\
\text { (pancreas) }\end{array}$ & - \\
\hline $\begin{array}{l}\text { Ear, nose, and } \\
\text { throat }\end{array}$ & - & - & $\begin{array}{l}\text { Squamous cell } \\
\text { carcinoma } \\
\left(\text { larynx/pharynx }{ }^{\mathrm{a}} \text { ) }\right.\end{array}$ & $\begin{array}{l}\text { Squamous cell } \\
\text { carcinoma }^{\text {(tongue }} \text { ) }\end{array}$ & - \\
\hline Gastrointestinal & - & - & $\begin{array}{l}\text { Sarcoma } \\
\text { (large intestine }^{a} \text { ) }\end{array}$ & $\begin{array}{l}\text { Lymphosarcoma } \\
\text { (small intestine }{ }^{\mathrm{a}} \text { ) }\end{array}$ & - \\
\hline $\begin{array}{l}\text { Hepatic and } \\
\text { biliary }\end{array}$ & - & $\begin{array}{l}\text { Adenoma } \\
\text { (liver) } \\
\text { Hepatoma } \\
\text { (liver) }\end{array}$ & $\begin{array}{l}2 \text { adenoma } \\
\text { (both liver) } \\
4 \text { carcinoma }^{\text {arcinoma }} \\
\text { (all liver }{ }^{\mathrm{a}, \mathrm{a}, \mathrm{a}} \text { ) } \\
\text { Unspecified } \\
\text { mass (liver }{ }^{\mathrm{a}} \text { ) }\end{array}$ & 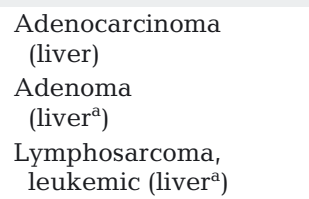 & - \\
\hline Immunologic & - & - & - & $\begin{array}{l}\text { Lymphoma } \\
\text { (spleen) } \\
2 \text { lymphosarcoma } \\
\text { (both spleen }{ }^{\mathrm{a}} \text { ) }\end{array}$ & - \\
\hline Integumentary & - & - & - & $\begin{array}{l}\text { Spindle cell } \\
\text { sarcoma (dorsum }{ }^{\mathrm{a}} \text { ) }\end{array}$ & - \\
\hline Musculoskeletal & - & - & $\begin{array}{l}\text { Adenocarcinoma } \\
\left(\text { skeletal muscle }^{\mathrm{a}}\right) \\
\text { Spinal mass }\end{array}$ & $\begin{array}{l}\text { Fibroma } \\
\text { (tarsal region) } \\
3 \text { fibrosarcoma } \\
\left(2 \text { lumbar }{ }^{\mathrm{a}, \mathrm{a}}, 1 \text { elbow }^{\mathrm{a}}\right) \\
\text { Osteosarcoma }\left(\text { (rib }^{\mathrm{a}}\right)\end{array}$ & - \\
\hline Ocular & - & Melanoma (iris) & - & - & - \\
\hline Renal & - & $\begin{array}{l}\text { Carcinoma } \\
\text { (kidney) }\end{array}$ & $\begin{array}{l}\text { Adenocarcinoma } \\
\left(\text { kidney }^{\mathrm{a}}\right) \\
\text { Carcinoma } \\
\text { (bladder }^{\mathrm{a}} \text { ) }\end{array}$ & $\begin{array}{l}\text { Leiomyosarcoma } \\
\left(\text { bladder }^{\mathrm{a}}\right) \\
\text { Spindle cell } \\
\left.\text { sarcoma (kidney }{ }^{\mathrm{a}}\right)\end{array}$ & $\begin{array}{l}\text { Adenocarcinoma } \\
\text { (kidney) }\end{array}$ \\
\hline Reproductive & $\begin{array}{c}\text { Granulosa cell } \\
\text { tumor (ovary) }\end{array}$ & - & $\begin{array}{l}2 \text { adenocarcinoma } \\
\left(1 \text { mammary, } 1 \text { uterus }^{\mathrm{a}}\right) \\
\text { Carcinosarcoma } \\
\left.\text { (uterus }^{\mathrm{a}}\right) \\
2 \text { leiomyoma } \\
\left.\text { (both uterus }^{\mathrm{a}}\right) \\
\text { Multiocular mass } \\
\text { (mammary) } \\
\text { Prostate mass } \\
\text { Uterine mass }^{\mathrm{a}}\end{array}$ & $\begin{array}{l}\text { Adenoma } \\
\text { (seminal vesicle) } \\
2 \text { carcinoma } \\
(1 \text { testis, } 1 \text { uterus) } \\
2 \text { leiomyoma } \\
\text { (both uterus }{ }^{\mathrm{a}} \text { ) } \\
\text { Granulosa cell } \\
\text { tumor (ovary) } \\
\text { Leydig cell } \\
\text { tumor (testis) } \\
2 \text { unspecified } \\
\text { testicular masses }\end{array}$ & $\begin{array}{l}\text { Carcinoma } \\
\left.\text { (endometrium }^{\mathrm{a}}\right)\end{array}$ \\
\hline Respiratory & $\begin{array}{l}\text { Carcinoma } \\
\text { (bronchioalveolar) }\end{array}$ & ) & $\begin{array}{l}2 \text { adenoma (both lung) } \\
\text { Carcinoma (lung) }\end{array}$ & Carcinoma (lung ${ }^{\mathrm{a}}$ ) & - \\
\hline Multi-systemic & - & - & $\begin{array}{l}\text { Unspecified mass } \\
\text { (abdominal) } \\
2 \text { adenocarcinoma } \\
2 \text { lymphosarcoma }{ }^{\mathrm{a}, \mathrm{a}} \\
\text { Sarcoma }^{\mathrm{a}} \\
\text { Transitional cell } \\
\text { carcinoma }\end{array}$ & $\begin{array}{l}\text { Unspecified mass } \\
\left(\text { abdominal }^{\mathrm{a}}\right) \\
\text { Lymphoma }^{\mathrm{a}} \\
\text { Lymphosarcoma } \\
\text { (leukemic) } \\
\text { Metastatic histiocytic } \\
\text { round cell tumor }^{\mathrm{a}}\end{array}$ & - \\
\hline
\end{tabular}


Table 6. Circumstances surrounding traumas related to death in lorises and pottos in North American facilities, 1980-2010; $(-)$ no cases reported

\begin{tabular}{|c|c|c|c|c|c|}
\hline Trauma type & $\begin{array}{l}\text { Loris tardigradus } \\
\text { nordicus }\end{array}$ & $\begin{array}{l}\text { Loris tardigradus } \\
\text { tardigradus }\end{array}$ & $\begin{array}{c}\text { Nycticebus } \\
\text { coucang }\end{array}$ & $\begin{array}{l}\text { Nycticebus } \\
\text { pygmaeus }\end{array}$ & $\begin{array}{l}\text { Perodicticus } \\
\text { potto }\end{array}$ \\
\hline $\begin{array}{l}\text { Bite wounds and } \\
\text { cannibalism }\end{array}$ & $\begin{array}{l}1 \text { neonate (housed } \\
\text { with dam and other } \\
\text { (?) cagemates, bite } \\
\text { wound and fall } \\
\text { during intragroup } \\
\text { aggression) }\end{array}$ & $\begin{array}{l}5 \text { neonates ( } 1 \text { from } \\
\text { conspecific neighbor, } \\
\text { others could have been } \\
\text { dam or sire) } \\
2 \text { juveniles ( } 1 \text { from } \\
\text { cagemate, other could } \\
\text { have been dam or sire) }\end{array}$ & $\begin{array}{l}5 \text { neonates ( } 1 \text { killed by } \\
\text { dam, } 2 \text { with suspect sire } \\
\text { or other male; } 2 \text { could } \\
\text { have been dam or sire) } \\
1 \text { juvenile (suspect } \\
\text { male cagemate) }\end{array}$ & $\begin{array}{l}6 \text { neonates } \\
\text { (1 suspect sire; } 1 \\
\text { conspecific neighbor; } \\
\text { housing situation } \\
\text { unclear for others) }\end{array}$ & 1 neonate \\
\hline $\begin{array}{l}\text { Infection/disease } \\
\text { secondary to } \\
\text { bite wound }\end{array}$ & - & $\begin{array}{l}1 \text { adult (septicemia) } \\
2 \text { geriatric (1 septicemia } \\
\text { from month-old bite } \\
\text { wound; } 1 \text { lung edema } \\
\text { possibly related to bite } \\
\text { wound thought to } \\
\text { have healed) }\end{array}$ & $\begin{array}{l}1 \text { juvenile (cellulitis and } \\
\text { necrosis on hands and } \\
\text { feet, bite from dam or } \\
\text { male neighbor (?)) } \\
3 \text { adults (1 severe } \\
\text { necrotizing myositis } \\
\text { of bite wound from } \\
\text { cagemate; } 1 \text { cellulitis } \\
\text { from cagemate bite; } 1 \text { had } \\
\text { chronic sinusitis resulting } \\
\text { from bite received as an } \\
\text { infant from dam) } \\
1 \text { geriatric (euthanized } \\
\text { for neoplasia, also had } \\
\text { other recent problems } \\
\text { including bite wounds) }\end{array}$ & $\begin{array}{l}1 \text { neonate } \\
\text { (septicemia from } \\
\text { hand mutilated by } \\
\text { mother) } \\
1 \text { juvenile (cellulitis, } \\
\text { history of chronic, poorly } \\
\text { healing bite wounds) } \\
1 \text { geriatric (purulent } \\
\text { abscess from bite } \\
\text { wound) } \\
1 \text { geriatric (infected } \\
\text { bite from armadillo) }\end{array}$ & - \\
\hline $\begin{array}{l}\text { Cases of scavenging } \\
\text { presumed to have } \\
\text { occurred postmortem }\end{array}$ & 1 neonate & 10 neonates & 1 neonate & 6 neonates & 2 neonates \\
\hline $\begin{array}{l}\text { Head trauma/ } \\
\text { traumatic fall }\end{array}$ & - & $\begin{array}{l}1 \text { neonate } \\
1 \text { adult (related } \\
\text { to metabolic } \\
\text { condition?) }\end{array}$ & - & $\begin{array}{l}4 \text { neonates } \\
\text { ( } 3 \text { incurred during } \\
\text { intragroup aggression) } \\
1 \text { juvenile (possible fall } \\
\text { related to maternal } \\
\text { neglect?) } \\
1 \text { geriatric (spinal } \\
\text { fracture, age-related?) }\end{array}$ & 2 neonates \\
\hline Other & - & - & $\begin{array}{l}1 \text { neonate (maternal } \\
\text { over-grooming) } \\
1 \text { adult } \\
\text { (interspecific predation) } \\
1 \text { adult (accident } \\
\text { involving cage } \\
\text { furnishings) }\end{array}$ & $\begin{array}{l}1 \text { adult with fetus } \\
\text { (interspecific predation) } \\
1 \text { geriatric (bite wounds } \\
\text { contributed to decision } \\
\text { to euthanize, cause } \\
\text { of death listed as renal } \\
\text { failure) }\end{array}$ & - \\
\hline
\end{tabular}

lopathy. There were no deaths attributed to neoplasia in this species, although some neoplastic growths were discovered in necropsy (Table 5).

The renal and cardiovascular systems were most commonly affected by disease in $L$. $t$. nordicus (Table 4). Animals suffering from renal disease had a group of diagnoses including nephritis, glomerulonephritis, fibrosis, renal infarcts, and distention. One case of cystitis was also included in the renal category. Cardiovascular and hemolymphatic diagnoses included cardiomyopathy, leukocytosis, fibrosis, myocarditis, endocardiosis, and epicarditis. CNS diseases included 2 cases of hemorrhage and notes of age-related histological changes in the brain. Ocular changes included synechiae, cataracts, blindness, and nerve fiber degeneration. Respiratory illnesses included pneumonia, serositis, mineralization, metaplasia, and 1 case of a benign bronchioalveolar adenocarcinoma. Whole-body changes of note included anorexia, serous atrophy of adipose tissue, and dehydration.

\section{Loris tardigradus tardigradus}

Cause of death in the red slender loris was most commonly attributed to multi-systemic disease (Table 3). Many of these deaths (5/14) were attrib- 
uted to septicemia, 1 to hepatitis secondary to bacterial infection, and 1 to infection-associated granulomatous lesions attacking multiple organ systems. In the remaining cases, animals were geriatric or had problems affecting multiple organ systems with no obviously primary condition. Deaths involving the renal system were also common, and were attributed generally to renal failure (6/13), fibrosis (1/13), or glomerulonephritis or nephritis (6/13).

Trauma and neonatal mortality were significant in this group as well (Table 6). Of the 10 deaths attributed to trauma, 2 were bite wounds (with subsequent infection in 1 case) that led to juvenile deaths, and 6 others were neonate deaths. Traumatic deaths in neonates occurred due to traumatic bone injury (1/9), aggression from a neighboring animal (1/9), and withingroup aggression leading to bite wounds and death (5/9). Additionally, 1 neonate death was attributed to maternal neglect and 14 were unexplained. Like $L$. $t$. nordicus, there were no deaths attributed primarily to neoplasia in L. t. tardigradus.

Overall, the most common pathologies affecting $L$. t. tardigradus occurred in the hepatic and biliary and respiratory systems (Table 4). Liver disease was common, and included cirrhosis, hepatitis, hyperplasia, congestion, fibrosis, lipidosis, vacuolar change and degeneration, and 1 presumptive case of hemosiderosis. Reports of liver neoplasias included 1 hepatoma and a hepatocellular adenoma. One animal reportedly suffered from an obstruction in the gall bladder and another from cholangitis. In the respiratory system, pneumonia was commonly reported. Other respiratory changes included atelectasis, hemorrhage, congestion, hemosiderosis, edema, fibrosis, and bronchitis. Cardiovascular issues included congestive heart failure, myocardial atrophy, fibrosis, myocarditis, and congestion. Vascular problems were also common, and included vasculitis, histiocytosis, arteriopathy, atherosclerosis, and thrombosis. Renal disease was a common finding, and in addition to the common renal changes listed above for $L$. $t$. nordicus, there was 1 report of a renal cyst and 1 of a renal cell carcinoma (Table 5).

\section{Nycticebus coucang}

Like the red slender loris, the most common causes of death for slow lorises were multi-systemic and renal diseases (Table 3). Neoplasia (Table 5) affecting multiple organ systems accounted for several (6/23) cases, and included 2 adenocarcinomas and 2 lymphosarcomas. Septicemia was responsible for 3 deaths and infections for 5 deaths. One animal reportedly died from toxicosis, but a toxin was never identified, and the remaining cases were all attributed to non-infectious disease processes (often agerelated) affecting multiple organ systems. Renal diseases included 2 neoplasias, cases described generally as renal failure (7/21), and nephritis, including pyelonephritis and glomerulonephritis (12/21).

After renal and multi-systemic disease, trauma was the most frequent cause of death in slow lorises (Table 6). Most victims of trauma (6/11) were neonates. One animal was euthanized due to pathological maternal over-grooming, and the others died following bite wounds and neglect from parents. Two juveniles died of trauma as a result of parental aggression: one from multiple skull fractures and the other from necrosis and cellulitis resulting from bite wounds. Three adults died as a result of traumatic events. One animal developed cellulitis secondary to wounds received from a conspecific, while another escaped an enclosure and was attacked by an ocelot. A 4 yr old male succumbed to acute heart failure following an immobilization made necessary because the loris had become entangled in a cargo net used as exhibit perching.

Neoplasia was common in slow lorises; including the cases above, $17.4 \%$ of animals (19/109) died or were euthanized due to neoplasia, meaning it was one of the leading causes of death in captive slow lorises (Tables $3 \& 5$ ). All 3 reproductive neoplasias affected females and were uterine in origin. Other notable causes of death were 2 probable cases of diabetes mellitus. Four animals died from focal infections: myocarditis, otitis, rhinitis, and a chronic abscess of the thigh muscle.

Overall, $>45 \%$ of slow lorises showed some lesions or abnormality in their hepatic and biliary, respiratory, and renal systems. Common liver diseases reported were hemosiderosis, hepatic lipidosis, hepatitis, and vacuolar change. The most common renal lesions were due to nephritis, glomerular or pyelonephritis. Diseases affecting the bladder occurred in 8 animals, including cystitis (6 cases), serositis, and 2 bladder cancers. Pneumonia was the most common respiratory finding, although several neoplasias were reported in this organ system as well (Table 5).

Several other organ systems showed a prevalence of disease of $>20 \%$. Cardiovascular disease noted at death included cardiomyopathy, fibrosis, endocardiosis, and myocarditis. Diseases of the endocrine and metabolic system targeted the adrenal glands (adrenalitis, amyloidosis, hemosiderosis), pancreas (mainly hyperplasia), and thyroid (goiters, cysts). The 
most common gastrointestinal diseases were enteritis and gastritis, in addition to parasitism in the gastrointestinal tract. Immunological changes noted at death included histiocytosis, hyperplasia, and neoplasia of the lymph nodes, and splenitis and congestion affecting the spleen. Nine animals showed evidence of hemosiderosis affecting the spleen and/or lymph nodes. Reproductive diseases affected females more than males and included cervicitis, pyometra, plancentitis, and ovarian cysts. Reproductive neoplasms occurred in several animals (Table 5), including cancers of the uterus, ovary, and prostate. Two geriatric animals, 1 male and 1 female, had mammary neoplasms. In general, cases of neoplasia were largely limited to geriatric animals.

\section{Nycticebus pygmaeus}

Neonatal mortality had a significant impact on the study group, accounting for $34.6 \%$ of deaths in our sample of pygmy slow lorises (Tables $2 \& 3$ ). Trauma $(11 / 46)$ and maternal neglect $(9 / 46)$ caused neonate deaths in $43.3 \%(20 / 46)$ of cases. When cases of unexplained neonate death that reference carcass scavenging are included, this total increases to $50 \%$. Traumas experienced by neonates included head trauma (4/11), inter-group aggression (1/11), and intragroup aggression (6/11). In several cases, neonates were cannibalized, and 1 infant was euthanized after losing several limbs. Four mature pygmy lorises died as a result of trauma. Two adults died from what was thought to be an acute toxicosis, but a toxin was never identified. One geriatric male died of septicemia following infection from an armadillo bite, and a 3 yr old gravid female was killed by an ocelot after escaping her home enclosure (Table 6).

The most common causes of death in pygmy slow lorises were renal and multi-systemic diseases. Renal diseases cited as the cause of death were renal failure (6/20), nephritis (10/20), and 1 case in which a kidney abscess led to peritonitis and eventual death. Two animals died from complications related to cystitis, which led to bladder necrosis in 1 case. Renal neoplasms were considered the primary cause of death for 2 animals (Table 5): 1 geriatric male (14 yr) developed a spindle cell sarcoma in the kidney, while an adult ( 7 yr) female was euthanized due to a leiomyosarcoma of the bladder. Three additional neoplasias were listed as multi-systemic deaths (3/19), all for geriatric animals. Other deaths coded as multisystemic included cases of septicemia (7/19), other infectious processes (5/19), and non-infectious dis- ease processes and aging (6/19). Neoplasias reported as the primary cause of death were found in nearly every organ system and accounted for $14.3 \%$ (19/ 133) of pygmy loris deaths overall (Table 5).

The organ systems most frequently affected at death for the pygmy loris are described in Table 4. Organ systems that showed pathology for $>20 \%$ of animals were the cardiovascular and hemolymphatic, gastrointestinal, hepatic and biliary, immunologic, renal, and respiratory systems. Neoplasia was common throughout all organ systems (Table 5).

\section{Perodicticus potto}

Most of the pottos in this study died as neonates (Tables $2 \& 3$ ). All reported cases of trauma involved neonates, and 2 of 7 unexplained neonate deaths involved scavenged bodies. Two additional neonate deaths were attributed to maternal neglect and 1 to a systemic infection. Additionally, 3 of 5 respiratory deaths occurred in neonates.

The majority of other deaths occurred in geriatric animals. Multi-systemic causes of geriatric death were all related to multiple organ failure with associated shock. Other geriatric deaths were attributed to cardiomyopathy, endocarditis, renal failure, and 1 endometrial carcinoma, representing the only death attributed to neoplasia in all pottos (Table 5).

Lesions occurring in $>20 \%$ of animals were found in the gastrointestinal, hepatic and biliary, renal, and respiratory systems (Table 4). Gastrointestinal pathologies included esophagitis, enteritis, and colitis. Liver conditions reported were cirrhosis, hepatitis, congestion, and necrosis. Most renal pathologies were varieties of nephritis; however, a geriatric male and female were both diagnosed with polycystic renal disease. The majority of respiratory disease cases were pneumonia. Other interesting lesions discovered at death included 4 animals with rhinitis and extensive arteriosclerosis in 3 animals.

\section{DISCUSSION}

We reviewed medical records for 367 lorises and pottos that died in North American zoos over a $30 \mathrm{yr}$ period. Our results clearly show that poor neonate survivorship is a major concern for individual health and welfare, as well as population management. However, animals that do survive the critical first month of life are likely to reach sexual maturity and live into the geriatric stage. Adult and geriatric ani- 
mals are most likely to die of renal disease or multisystemic issues such as systemic infections, neoplasia, or multiple organ failure. High neonate mortality was due to high stillbirth percentages as well as trauma, which was often inflicted by conspecifics. Adults also fall victim to trauma from cagemates, suggesting that greater efforts to address the social management of lorises in captivity would likely have positive impacts on population sustainability and animal health.

Other studies have also found exceptionally high infant mortality in lorisiform primates living in both zoo and sanctuary settings. Streicher (2004) summarized health problems experienced by pygmy lorises confiscated from the wildlife trade in Vietnam. Of the 15 deaths reported over an 8 yr period, 11 were animals $<1 \mathrm{yr}$ old; overall, $42 \%$ of the sanctuary animals died during their first year of life. Debyser (1995) found infant mortality to be higher among lorises and their close relatives compared to other strepsirrhines, and like others (Tartabini 1991, Streicher 2004), Debyser (1995) speculated that captivity-induced stress was likely an underlying cause of infant deaths involving maternal neglect and trauma.

Information about social conditions was difficult to infer from our medical records, but it was clear that infants in this group were killed by sires, dams, and other conspecifics. Several different fitness benefits have been hypothesized to explain infanticide among nonhuman primates, including male-male competition and resource competition (Hrdy 1979). Infanticide risk may be related to social density, which is interesting considering that cage sizes for captive pottos and slender lorises in North America are currently smaller than the minimum size recommended by Fitch-Snyder \& Schulze's (2001) husbandry manual (Fuller et al. 2013). Deaths involving maternal neglect in the present study hint at a pattern similar to that described for captive Galago crassicaudatus umbrosus by Tartabini (1991). Failure to perform maternal behavior, for example due to poor socialization or stress related to the captive setting, leads to infant starvation and death, and the dead infant becomes a resource to consume (Tartabini 1991). If this is the case, then careful attention to postpartum maternal behavior and, if necessary, swift intervention may be important for saving infant lives. Maternal parity and litter sizes are also associated with infant survivorship (Pollock 1986), and new mothers or multiple births will likely require special attention.

Management of group composition around the perinatal period is likely to have a large impact on infant survivorship. Nekaris (2003) speculates that paternal care may be one factor promoting the relatively greater fecundity of slender than slow lorises, and paternal care has also been observed in captive pottos (Frederick 1998). These kinds of socialization opportunities are important for the formation and maintenance of social groups, and the 2001 husbandry manual states that it is preferable to keep groups intact through births unless there is cause to suspect an individual needs to be removed (FitchSnyder \& Schulze 2001). However, it is possible that social management practices surrounding parturition have changed in recent years, perhaps to address traumatic infant death. A 2010 survey of AZA facilities housing lorisids showed that very few males and females were housed together during the period surrounding birth, although more facilities indicated that they had attempted this strategy in the past (Fuller et al. 2013). That survey also showed that a large number of animals in each species were solitarily housed on a perpetual basis (Fuller et al. 2013). It may be that efforts to increase the social wellbeing of captive lorisids by providing social partners may be in conflict with strategies needed to minimize infant mortality. Given the tenuous status of these captive populations, isolating gravid females is probably a sound strategy until the proximate causes underlying infanticide are better understood.

It was often not possible in our study to distinguish between cases of infanticide, neglect, or trauma, or other possible causes of death, because infant carcasses were cannibalized. Although recovery of nutritional resources is a possible explanation for cannibalism, this behavior has been rarely observed in wild primates; if cannibalism is truly more prevalent in captive and reintroduced animals, then it is likely pathological in nature (Tartabini 1991, Dellatore et al. 2009). However, lack of tissues for necropsy renders it impossible to determine if infants actually died due to congenital or other disease states, or if infants were neglected by mothers because they were weak or ill. Greater efforts should be made to promptly remove deceased infants so that postmortem exams can clarify these issues.

Traumas were a significant contributor to mortality of adult as well as immature animals. In several cases, animals died following bite wounds that were chronically non-healing, leading to necrosis and cellulitis. In one case, a 3 yr old adult slow loris died from chronic sinusitis from an infection that originated with a bite wound sustained from his mother as a neonate. It has been speculated that a chemical produced by the brachial gland, mixed with slow loris saliva, may be the source of toxicity in bites 
(Krane et al. 2003). In addition to a major peptide component homologous to the cat allergen Fel-d1, loris venom contains a number of volatile compounds that suggest it plays a role in communication as well as anti-predator defense (Hagey et al. 2007). Necrotic bite wounds have also been reported in sanctuaryhoused pygmy lorises (Streicher 2004), and more information on the chemical structure and physiological role of loris venom may aid caretakers in the treatment and management of wounds.

Across the species in the present study, renal disease was the second most common cause of death, and the renal system exhibited the greatest frequency of lesions at death compared to all other organ systems. Renal changes such as glomerulosclerosis and nephritis are known to be prevalent in captive strepsirrhines and are often age-related (Burkholder 1981, Fitch-Snyder \& Schulze 2001, Junge 2003). Renal disease appears to occur more frequently in lorisoids (lorises, pottos, and galagos) than lemur species (Boraski 1981). Iron storage disease, or hemosiderosis, is a common pathology in lemurs (Benirschke et al. 1985), but was not very prevalent in the lorises and pottos in the present study.

A review of necropsies of Loris tardigradus housed at a German university revealed several cases of cholelithiosis or gallstones, all of which were composed of cholesterol and were speculated to be related to dietary factors like the presence of egg yolk and the reduced insect composition in the captive diet (Plesker \& Schulze 2006). Of the 2 cases of gallstones in the present study, 1 occurred in $L$. $t$. tardigradus and 1 in $L$. $t$. nordicus, but none were reported for slow lorises or pottos. We also recorded 1 case of diabetes mellitus in L. t. tardigradus, 5 in $\mathrm{Nyc}$ ticebus coucang, and 2 in N. pygmaeus. Captive lorises are prone to obesity (Ratajszczak 1998, FitchSnyder \& Schulze 2001), but body condition was not reported consistently at death, so its role in the development of degenerative diseases is not evident here.

It seems likely that diet is a contributing factor to non-infectious and degenerative diseases in captive lorises. The low metabolic rate characteristic of lorises is associated with a natural diet high in toxic secondary compounds from insects and plants (Wiens et al. 2006). It is tempting to speculate that metabolic derangement may occur when a diet this specialized cannot be replicated in the captive setting. The Mysore slender loris Loris lydekkerianus lydekkerianus feeds almost exclusively on animal prey, many of which are toxic species (Nekaris \& Rasmussen 2003). New data from the field has highlighted the importance of nectars and exudates in the slow loris diet (Tan \& Drake 2001, Starr \& Nekaris 2013); at one study site in India, $N$. bengalensis fed almost exclusively on exudates during the winter season (Swapna et al. 2010). Lorises often obtain gums by gouging at trees, and the lack of opportunities to perform this behavior in captivity may be associated with periodontal disease (Nekaris 2009).

We were surprised by how rarely dental disease was indicated on histopathology and necropsy reports of the animals we studied. It seems likely that in this study, many necropsy reports did not include notes of missing teeth or gingival disease that may have occurred many years earlier and/or seemed unrelated to the immediate cause of death. Dental disease has previously been reported as a major source of morbidity for Asian lorisines in captivity (Sutherland-Smith \& Stalis 2001). In a sample of 25 Loris tardigradus at a German university, 7 individuals had missing or loose teeth, or severe calculus at death, and another 4 animals showed evidence of inflammation and infection secondary to dental disease (Plesker \& Schulze 2013). In the present study, several cases were noted in which a retrobulbar or other facial abscess occurred secondary to dental infection. Dental disease may play an under-recognized role in loris health, and more efforts should be made to institute preventative care during routine exams (Plesker \& Schulze 2013) and to identify dental abnormalities or infections at necropsy.

Around $10 \%$ of the animals in our sample died as a result of neoplasia. A malignant lymphoma that occurred in a slow loris in this sample has been previously described elsewhere and was thought to be caused by a herpes viral infection (Stetter et al. 1995). Our sample also overlaps with neoplasias reported for the Duke Lemur Center (DLC), which contributed records to this study, and at which cases of neoplasia have been thoroughly described (Remick et al. 2009, Zadrozny et al. 2010). However, cases of hepatic neoplasia were reported from 4 additional facilities in the present study, suggesting liver cancer may play an important role in mortality for AZA loris populations as a whole. Our results concur with those reviewed by Remick et al. (2009), that neoplasia in lorises and pottos, like other strepsirrhines, commonly occurs in the digestive (including liver), hematopoietic (here, these were generally classified as multi-systemic), and reproductive systems. Little research has addressed the etiology of cancers affecting captive strepsirrhines, and further research into the role of diet (Bingham et al. 1976, Cowgill et al. 1989), exhibit lighting (Navara \& Nelson 2007, Fuller et al. 2013), and other factors contributing to neoplasia is needed. 


\section{CONCLUSIONS}

After reviewing death records spanning $30 \mathrm{yr}$ for all lorises and pottos in North American zoos, it is clear that social management remains a challenge in these species. Deaths due to trauma are common and most likely to affect neonates and juveniles before they have the opportunity to reproduce. These trends suggest that targeting efforts to improve infant survival could have major benefits for the sustainability of captive populations. Animals that live to adulthood are likely to die as a result of infectious agents, neoplasia, or degenerative changes. Renal disease remains a major source of pathology for all lorises and pottos, and further investigation is needed to understand its etiology. A greater understanding of the role of diet in the development of non-infectious disease is also needed.

Acknowledgements. We thank the following facilities for contributing medical records to this study: Albuquerque Biological Park; Aquarium \& Rainforest at Moody Gardens; The Calgary Zoo, Botanical Garden \& Prehistoric Park; Chicago Zoological Society-Brookfield Zoo; Cincinnati Zoo \& Botanical Garden; Cleveland Metroparks Zoo; Columbus Zoo and Aquarium; Denver Zoo; Detroit Zoo; Duke Lemur Center; El Paso Zoo; Franklin Park Zoo; Houston Zoo, Inc.; Lake Superior Zoological Gardens; Lee Richardson Zoo; Lincoln Park Zoo; Los Angeles Zoo and Botanical Gardens; The Maryland Zoo in Baltimore; Memphis Zoo; Mesker Park Zoo \& Botanic Garden; Minnesota Zoological Garden; Oglebay's Good Zoo; Omaha's Henry Doorly Zoo; The Philadelphia Zoo; San Antonio Zoo; San Diego Zoo and San Diego Wild Animal Park; San Francisco Zoo; Santa Ana Zoo; Trevor Zoo; Virginia Zoological Park; Wildlife Conservation Society - Bronx Zoo; and Woodland Park Zoo. Several individuals went above and beyond to accommodate this request, including Julie Parks Taylor, Jenn Harrison, Jeannine Jackle, Andrea Katz, and Mark Campbell. We are also grateful to the many volunteers who assisted with data entry, including Gail Simpson, Jessica Taylor, Alyssa Mills, and Lauren Starkey. We also thank Anna Nekaris for inviting us to submit our paper to this Theme Section on slow loris ecology and conservation, and Kym Gopp for her support of this project.

\section{LITERATURE CITED}

Benirschke K, Miller C, Ippen R, Heldstab A (1985) The pathology of prosimians, especially lemurs. Adv Vet Sci Comp Med 30:167-208

Bingham G, Sembrat R, Migaki G (1976) Myeloliposarcoma in a Perodicticus potto-case report. Lab Anim Sci 26: 473-477

- Boraski EA (1981) Renal disease in prosimians. Vet Pathol 18:1-5

Brandon-Jones D, Eudey AA, Geissmann T, Groves CP and others (2004) Asian primate classification. Int J Primatol 25:97-164
Burkholder PM (1981) Glomerular disease in captive galagos. Vet Pathol 18:6-22

Charles-Dominique P (1977) Ecology and behaviour of nocturnal primates. Columbia University Press, New York, NY

Cowgill UM, States SJ, States KJ (1989) A twenty-five-year chronicle of a group of captive nocturnal prosimians (Perodicticus potto). Mammal Rev 19:83-89

$>$ Debyser IWJ (1995) Prosimian juvenile mortality in zoos and primate centers. Int J Primatol 16:889-907

Dellatore DF, Waitt CD, Foitova I (2009) Two cases of motherinfant cannibalism in orangutans. Primates 50:277-281

Fitch-Snyder H, Schulze H (2001) Management of lorises in captivity: a husbandry manual for Asian lorisines (Nycticebus and Loris spp.). Zoological Society of San Diego, San Diego, CA

Frederick C (1998) Observations of paternal care in Perodicticus potto at the Cincinnati Zoo and Botanical Garden. Folia Primatol (Basel) 69:312-317

> Fuller G, Kuhar CW, Dennis PM, Lukas KE (2013) A survey of husbandry practices for lorisid primates in North American zoos and related facilities. Zoo Biol 32:88-100

> Grubb P, Butynski TM, Oates JF, Bearder SK, Disotell TR, Groves CP, Struhsaker TT (2003) Assessment of the diversity of African primates. Int J Primatol 24:1301-1357

Hagey LR, Fry BG, Fitch-Snyder H (2007) Talking defensively: a dual use for the brachial gland exudate of slow and pygmy lorises. In: Gursky S, Nekaris KAI (eds) Primate anti-predator strategies. Springer, New York, NY, p 251-270

Hope K, Deem SL (2006) Retrospective study of morbidity and mortality of captive jaguars (Panthera onca) in North America: 1982-2002. Zoo Biol 25:501-512

Hrdy SB (1979) Infanticide among animals: a review, classification, and examination of the implications for the reproductive strategies of females. Ethol Sociobiol 1: $13-40$

Iseborn T, Starr CR, Nekaris KAI (2011) Field surveys and local knowledge highlight threatened status of Cambodia's slow lorises (Nycticebus pygmaeus and N. bengalensis). Folia Primatol (Basel) 82:388-389

Junge ER (2003) Prosimians. In: Fowler ME, Miller RE (eds) Zoo and wild animal medicine. Saunders, Philadelphia, PA, p 334-346

> Krane S, Itagaki Y, Nakanishi K, Weldon PJ (2003) 'Venom' of the slow loris: sequence similarity of prosimian skin gland protein and Fel d 1 cat allergen. Naturwissenschaften 90:60-62

Kuhar C, Fitch-Snyder H, Arnold K, Buckanoff H and others (2011) Population analysis and breeding and transfer plan: nocturnal prosimian programs. Lincoln Park Zoo, Chicago, IL

> Munds RA, Nekaris KAI, Ford SM (2013) Taxonomy of the Bornean slow loris, with new species Nycticebus kayan (Primates, Lorisidae). Am J Primatol 75:46-56

> Navara KJ, Nelson RJ (2007) The dark side of light at night: physiological, epidemiological, and ecological consequences. J Pineal Res 43:215-224

Nekaris KAI (2003) Observations of mating, birthing and parental behaviour in three subspecies of slender loris (Loris tardigradus and Loris lydekkerianus) in India and Sri Lanka. Folia Primatol (Basel) 74:312-336

Nekaris KAI (2009) Comparative ecology of exudate feeding by Asian lorises (Loris, Nycticebus). Am J Phys Anthropol Suppl 48:199 
Nekaris KAI, Campbell N (2012) Media attention promotes conservation of threatened Asian slow lorises. Oryx 46: 169-170

Nekaris KAI, Jaffe S (2007) Unexpected diversity of slow lorises (Nycticebus spp.) within the Javan pet trade: implications for slow loris taxonomy. Contrib Zool 76:187-196

> Nekaris KAI, Jayewardene J (2004) Survey of the slender loris (Primates, Lorisidae Gray, 1821: Loris tardigradus Linnaeus, 1758 and Loris lydekkerianus Cabrera, 1908) in Sri Lanka. J Zool 262:327-338

Nekaris KAI, Nijman V (2007) CITES proposal highlights rarity of Asian nocturnal primates (Lorisidae: Nycticebus). Folia Primatol (Basel) 78:211-214

Nekaris KAI, Rasmussen DT (2003) Diet and feeding behavior of Mysore slender lorises. Int J Primatol 24:33-46

Nekaris KAI, Shepherd CR, Starr CR, Nijman V (2010) Exploring cultural drivers for wildlife trade via an ethnoprimatological approach: a case study of slender and slow lorises (Loris and Nycticebus) in South and Southeast Asia. Am J Primatol 72:877-886

Plesker R, Schulze H (2006) Polycystic nephropathy in slender lorises (Loris lydekkerianus). Am J Primatol 68: 838-844

Plesker R, Schulze H (2013) Dental disease in slender lorises (Loris tardigradus). Zoo Biol 32:571-574

Pollock JI (1986) The management of prosimians in captivity for conservation and research. In: Benirschke K (ed) Primates: the road to self-sustaining populations. SpringerVerlag, New York, NY, p 269-288

Ratajszczak R (1998) Taxonomy, distribution and status of the lesser slow loris Nycticebus pygmaeus and their implications for captive management. Folia Primatol (Basel) 69:171-174

Remick AK, Van Wettere AJ, Williams CV (2009) Neoplasia in prosimians: case series from a captive prosimian pop-

Editorial responsibility: Anna Nekaris,

Oxford, UK ulation and literature review. Vet Pathol 46:746-772

Schulze H (1998) A 'checklist' of possible items for prosimian husbandry manuals and research. Folia Primatol (Basel) 69:152-170

> Starr C, Nekaris KAI (2013) Obligate exudativory characterizes the diet of the pygmy slow loris Nycticebus pygmaeus. Am J Primatol 75(10):1054-1061

Stetter MD, Worley MB, Ruiz B (1995) Herpesvirus-associated malignant lymphoma in a slow loris (Nycticebus coucang). J Zoo Wildl Med 26:155-160

Streicher U (2004) Aspects of ecology and conservation of the pygmy loris Nycticebus pygmaeus in Vietnam. PhD dissertation, Ludwig-Maximilians Universität München

Sutherland-Smith M, Stalis I (2001) Review of loris clinical information and pathological data from the San Diego Zoo 1982-1995. In: Fitch-Snyder H, Schulze H (eds) Management of lorises in captivity: a husbandry manual for Asian lorisines (Nycticebus and Loris spp.). Zoological Society of San Diego, San Diego, CA, p 60-70

> Swapna N, Radhakrishna S, Gupta AK, Kumar A (2010) Exudativory in the Bengal slow loris (Nycticebus bengalensis) in Trishna Wildlife Sanctuary, Tripura, Northeast India. Am J Primatol 72:113-121

Tan CL, Drake JH (2001) Evidence of tree gouging and exudate eating in pygmy slow lorises (Nycticebus pygmaeus). Folia Primatol (Basel) 72:37-39

> Tartabini A (1991) Mother-infant cannibalism in thick-tailed bush-babies (Galago crassicaudatus umbrosus). Primates 32:379-383

> Wiens F, Zitzmann A, Hussein NA (2006) Fast food for slow lorises: Is low metabolism related to secondary compounds in high-energy plant diet? J Mammal 87:790-798

> Zadrozny LM, Williams CV, Remick AK, Cullen JM (2010) Spontaneous hepatocellular carcinoma in captive prosimians. Vet Pathol 47:306-311

Submitted: June 27, 2013; Accepted: November 11, 2013 Proofs received from author(s): March 4, 2014 